\title{
X-Ray Mapping of an Impact-Resistant Crustacean-Derived Biocomposite
}

Nicholas A. Yaraghi ${ }^{1}$, Nicolás Guarín-Zapata ${ }^{2}$, Eric Hintsala ${ }^{3}$, Sanjit Bhowmick $^{4}$, Leigh Sheppard ${ }^{5}$, Pablo D. Zavattieri ${ }^{2}$, Richard Wuhrer ${ }^{6}$, and David Kisailus ${ }^{1,7}$

1. Materials Science \& Engineering, University of California, Riverside, Riverside, California, USA

2. Lyles School of Civil Engineering, Purdue University, West Lafayette, Indiana, USA

3. Chemical Engineering and Materials Science, University of Minnesota, Minneapolis, Minnesota, USA

${ }^{4 .}$ Hysitron Inc., Minneapolis, Minnesota, USA

5. School of Computing, Engineering and Mathematics, Western Sydney University, Penrith, Australia

6. Advanced Materials Characterization Facility, Western Sydney University, Penrith, Australia

7. Chemical and Environmental Engineering, University of California, Riverside, California, USA

Nature is renowned for its ability to synthesize composite materials, under relatively mild conditions of temperature, pressure, and $\mathrm{pH}$, that exhibit a high degree of sophistication and complexity. Biomineralized tissues are one such class of materials, which feature organic and inorganic constituents that are carefully arranged over multiple length-scales to produce hierarchical structures exhibiting exceptional mechanical properties such as strength and toughness. One notable example is the raptorial appendage (dactyl club) of the mantis shrimp (Figure 1a), a group of aggressive marine crustaceans. This hammer-like club (Figure 1b) is used to smash through the tough exoskeletal structures of mollusks, crustaceans, and other shelled prey with incredible force (up to $1500 \mathrm{~N}$ ) and speed (23 m/s from rest), demonstrating one of the fastest striking events found in Nature [1].

The success of the dactyl club in resisting catastrophic failure from repetitive high energy impacts lies in its multi-regional and multi-functional composite architecture, featuring an organic matrix composed of sheet-like and helicoidally-arranged alpha-chitin fibers mineralized by amorphous forms of calcium carbonate and calcium phosphate as well as highly textured crystalline apatite [2]. Here we use energy dispersive spectroscopy (EDS) and x-ray mapping (XRM) techniques to identify elemental features and compositional gradients within the stomatopod dactyl club [3]. This data is then correlated with ultrastructural and mechanical analyses to not only derive structure-property relationships, but also glean insight into the assembly and biomineralization process.

Figure 1c shows an optical micrograph of a polished cross-section of the club, highlighting its multiregional nature. XRM (Figures 1d-f), showing the local distribution of calcium, phosphorus, and magnesium, respectively, reveal the high degree of mineralization, with the highest concentrations of calcium and phosphorus localized in outer surface (impact region) corresponding to an apatitic mineral [2]. Magnesium, concentrated within the interior (periodic region) of the club, plays an important role in stabilizing the amorphous calcium carbonate and calcium phosphate phases present. Figure $2 \mathrm{a}$ shows a pseudo-colored micrograph of the dactyl club cross-section revealing local distribution of calcium (red), phosphorus (green), and sulfur (blue). Scatter diagrams of calcium vs. phosphorus, calcium vs. sulfur, and phosphorus vs. sulfur (Figures $2 \mathrm{~b}-\mathrm{d}$, respectively) reveal nodes, which correspond to unique compositions within the dactyl club, identified as the impact region, the interface between the impact and periodic regions, the medial periodic region, and the lateral periodic region (Figure 2e). Interestingly, these elemental phases correlate well with regional variations in the local microstructure and mechanical properties, as identified by high resolution electron microscopy and nanomechanical 
testing. These aspects, which have implications for imparting strength and fracture tolerance to the dactyl club, will be discussed in more detail in this talk [4].

\section{References:}

[1] S. N. Patek, R. L. Caldwell, Journal of Experimental Biology 208 (2005), pp. 3655-3664.

[2] J. Weaver et al., Science 336 (2012), pp.1275-1280.

[3] K. Moran, R. Wuhrer, Microchimica Acta 155 (2006), pp. 209-217.

[4] This work was supported by AFOSR-FA9550-12-1-0245 and AFOSR-FA9550-15-1-0009.
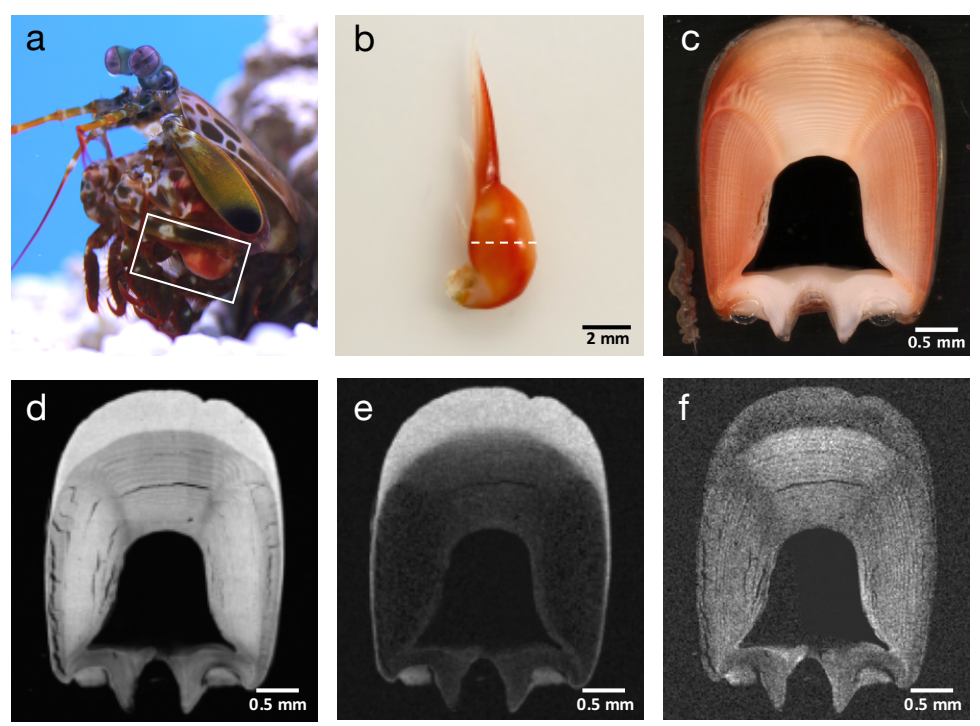

Figure 1. (a) Anterior of $O$. scyllarus with the dactyl club highlighted in white; optical micrographs showing (b) the dactyl segment and (c) polished cross-section corresponding to the dashed line in (b); (d-f) x-ray maps showing distributions of magnesium, calcium and phosphorus, respectively.
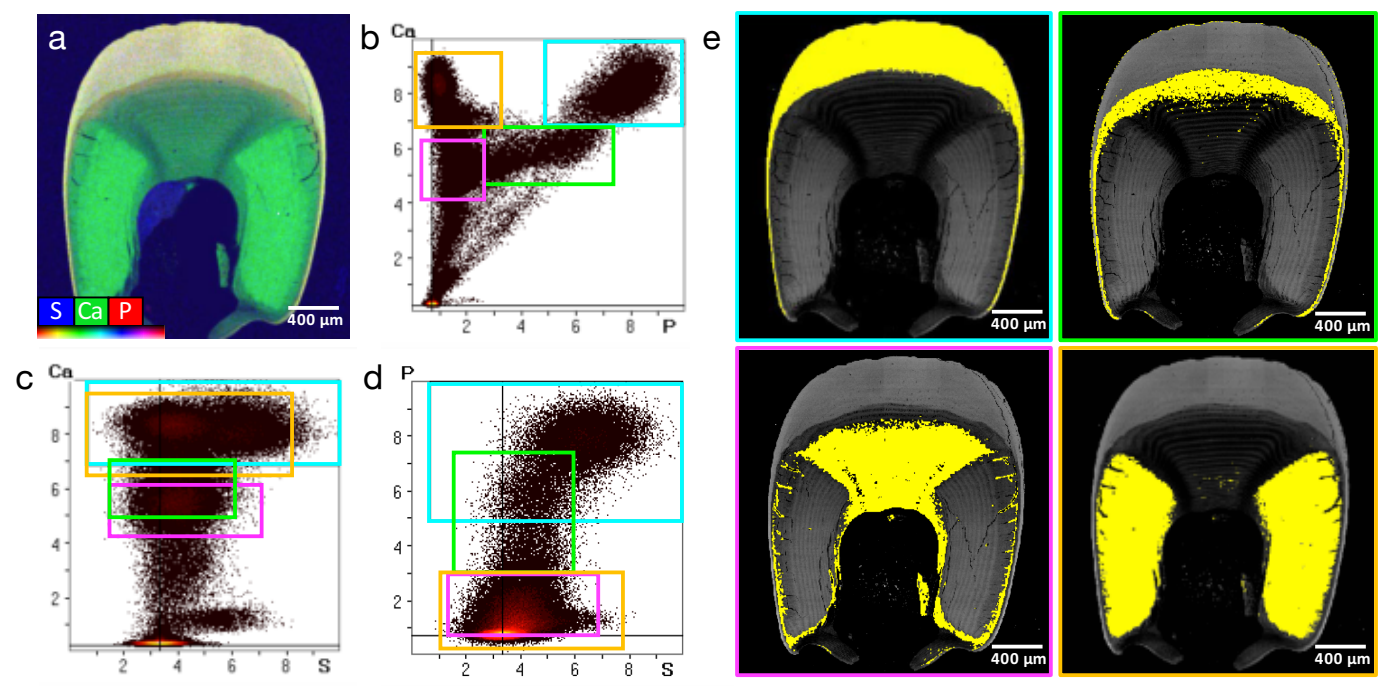

Figure 2. (a) Pseudo-colored micrograph of the dactyl cross-section showing concentrations of sulfur, calcium, and phosphorus. (b-d) Scatter diagrams of (b) calcium vs. phosphorus, (c) calcium vs. sulfur, and (d) phosphorus vs. sulfur; (e) Micrographs highlighting (in yellow) areas of unique composition corresponding to boxed regions of the scatter diagrams. 